\title{
Original Research \\ The utility of radiographic assessment of the internal mammary arteries in chest wall irradiated patients
}

\author{
Vamsidhar Naraparaju ${ }^{1,2, \dagger}$, Wassim Mosleh $^{2, \dagger}$, Mansour Almnajam², Mansour Khaddr ${ }^{2}$, \\ Swapnil Bagade $^{3}$, Anthony Posteraro ${ }^{3}$, David Grew ${ }^{4}$, Dorothy B. Wakefield ${ }^{1}$, David J. Hur ${ }^{5,6}$, \\ Aseem Vashist ${ }^{1,2,5, *}$ \\ ${ }^{1}$ Division of Cardiology, Hoffman Heart and Vascular Institute, Saint Francis Hospital, Hartford, CT 06105, USA \\ ${ }^{2}$ Division of Cardiology, University of Connecticut, Farmington, CT 06030, USA \\ ${ }^{3}$ Division of Radiology, Saint Francis Hospital, Hartford, CT 06105, USA \\ ${ }^{4}$ Division of Radiation-Oncology, Saint Francis Hospital, Hartford, CT 06105, USA \\ ${ }^{5}$ Division of Cardiology, VACT Healthcare System, CT 06111, USA \\ ${ }^{6}$ Section of Cardiovascular Medicine, Yale School of Medicine, New Haven, CT 06510, USA \\ *Correspondence: vashist@uchc.edu (Aseem Vashist) \\ ${ }^{\dagger}$ These authors contributed equally. \\ Academic Editor: Arthur J. Chu \\ Submitted: 11 September 2021 Revised: 29 November 2021 Accepted: 8 December 2021 Published: 18 January 2022
}

\begin{abstract}
Purpose: The internal mammary arteries (IMA's) are historically recognized to be protected against atherosclerosis. Whether chest wall-irradiation for breast cancer leads to significant IMA damage remains unclear. The utility of computed tomography (CT) and mammography to detect radiation-induced damage to the IMA's and its branches is not known. The objective of this study is to assess the susceptibility of IMA's to radiation-induced atherosclerosis, and the utility of CT scan and mammography in the assessment of IMA and its branches. Methods: A retrospective analysis of breast cancer patients who received chest wall-radiotherapy was performed. Patients with CT scans and/or mammograms $\geq 5$ years post-radiotherapy were included. Baseline characteristics, coronary artery calcification (CAC), the presence of IMA damage assessed by CT scan, and IMA branch calcifications by mammography were recorded. Results: None of the 66 patients with CT scans post-radiotherapy revealed IMA atherosclerosis. There were 28 (42.4\%) patients with CAC, of which four (14.3\% of CAC subgroup or $6.1 \%$ of the total cohort) had calcifications on either side on mammogram (Chi-square test, $p=$ 0.74). Out of the 222 patients with mammograms, 36 (16.2\%) had IMA branch calcifications. Two hundred and ten patients received unilateral radiotherapy, and 27 (12.9\%) of these patients had calcifications on the irradiated side, and 26 patients $(12.4 \%)$ had calcifications on the contralateral side $(\mathrm{OR}=1.0)$. Conclusion: IMA's do not exhibit signs of radiation-induced atherosclerosis when evaluated by $\mathrm{CT}$ scan. In addition, there is no association between radiotherapy for breast cancer and the presence of IMA branch calcification on mammograms.
\end{abstract}

Keywords: Internal mammary artery; CT scan; Mammogram; Calcification; Radiotherapy; Breast cancer

\section{Introduction}

An expanded role for radiation therapy in the management of breast cancer has come at the cost of radiationinduced coronary artery disease (RICAD). Compared to non-irradiated patients, patients who underwent chest wallradiotherapy for breast cancer have a higher absolute risk of cardiac morbidity and death, with the increase in risk being proportional to the radiation dose [1]. Ionizing-radiation induces reactive oxygen species resulting in accelerated atherosclerosis by endothelial damage and the impaired ability to clear free radicals [2], which leads to a chronic inflammatory state [3]. Atherosclerotic lesions in RICAD are more proximal than those in non-irradiated vessels and the plaques are largely composed of fibrous tissue [4]. In left-sided breast cancer, the anterior and apical wall of the heart, and the internal mammary arteries (IMA) can be in the radiation field.
The frequency of IMA graft failure after coronary artery bypass grafting (CABG) is common (8.6\%) [5], and the question of whether chest wall irradiation for the treatment of breast cancer leads to IMA damage remains unclear. Despite limited evidence, careful angiographic or radiographic evaluation and inspection of the IMA's before grafting has been recommended [6-8]. However, there is a paucity of data demonstrating radiographically that the IMA can be affected by radiation, or if it is inherently protected against atherosclerosis.

The present study aims to assess the utility of radiographic assessment of the IMA's and its branches by CT scan and mammography. 


\section{Materials and methods}

\subsection{Setting and design}

This is a retrospective cross-sectional analysis conducted at Saint Francis Hospital and Medical Center, a 617bed, urban tertiary care hospital in Hartford, CT. The hospital Institutional Review Board approved the study. After screening 1292 patients with breast cancer who received chest wall-radiotherapy at the single center within the study period, a total of 256 patients with a follow up imaging study at least 5 years or later from the date of initial radiation were included in the analysis. Inclusion was limited to breast cancer patients as opposed to other mediastinal cancers to preserve the homogeneity of radiation therapy protocols and dosing within the cohort.

All identifiable patients with breast cancer who received chest wall-radiotherapy from January 1st, 2003 to January 1st, 2014, were screened by chart review of electronic medical records. Patients with available chest CT scans and/or mammograms $\geq 5$ years post-radiotherapy were included. Patients who had IMA grafting were excluded. Patients were included in either the CT analysis, mammogram analysis, or both, depending on test availability for a given patient (Fig. 1). Data abstraction was performed independently by two abstracters (VN and MA). For each patient, the baseline demographics, cardiovascular risk factors, laboratory data, concomitant chemotherapy, mastectomy, and radiation therapy characteristics were collected by chart review.

\subsection{Image acquisition and analysis}

Baseline (pre-radiation therapy) CT scan, and the latest CT scan (post-radiation therapy), along with the latest mammogram (post-radiation therapy) were independently interpreted by two cardiac radiologists (SB and AP). The readers were blinded to the laterality of breast cancer or radiation therapy. Any discrepancies were settled by joint review discussions and consensus.

\subsection{Mammography}

Surveillance mammograms were obtained with a Hologic scanner in craniocaudal and mediolateral-oblique projections. For mammograms, craniocaudal projections for each breast were evaluated for the presence of tram track vascular calcifications. The vascular calcifications in the breasts on mammograms are Monckeberg calcifications from medial arteriosclerosis [9]. The IMA's supply the medial or central aspect of the breast parenchyma [10]. Therefore, calcifications found medial to the midline of the breast parenchyma were considered to be within the IMA supply territory.

\subsection{Computed tomography}

For chest CT scans, eighteen CT angiograms (CTA and CCTA), twelve contrast-enhanced CT scans (CECT), thirty-three non-contrast CT scans and, three CT scans with

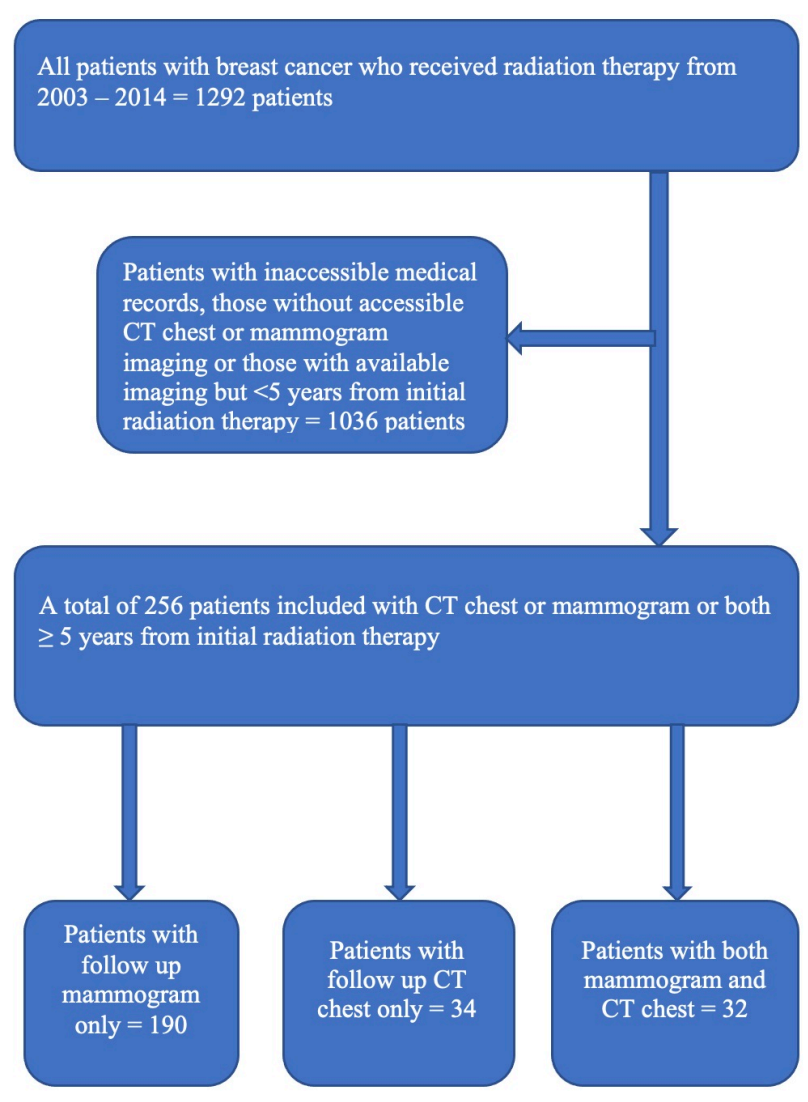

Fig. 1. Study design including screening and inclusion process by imaging modality.

and without contrast enhancement were included for analysis. Calcium scoring CT scans were not included as the entire extent of IMA's was not imaged by them. CT scans were obtained on Siemens Flash dynamic 256-detector CT and Siemens Definition 128-slice and Siemens Somatom 16-slice scanners. CECT and CT angiograms (CTA and CCTA) were obtained with an injection of 70 to $120 \mathrm{~mL}$ of Isovue 370 iodinated contrast. For CCTAs, electrocardiogram (ECG) gating was used.

Qualitative evaluation of the CT scans in trans-axial planes was performed for the presence of radiographicallydetected damage to the IMA's including the presence of IMA calcifications, patency, and occlusion/atresia. The presence of coronary calcifications was also recorded from the scans when assessable even on the non-gated studies and contrast-enhanced studies. IMA's were evaluated visually for calcifications. IMA patency was defined as a contrast column in the lumen as assessed by CTA or CECT. IMA occlusion or atresia was defined as non-visualization or an abrupt change in the vessel diameter at any site along its course in comparison to a proximal reference patent segment, as assessed by CTA or CECT. If streak or motion artifacts limited the evaluation, the CT was excluded from the analysis. Coronary calcifications if detected on visual interpretation were also recorded. No software calculations 
for calcium scoring were used. Patients with only CTA and CCTA had no pre-contrast imaging to accurately identify coronary calcifications. However, the presence or absence of calcifications was documented based on qualitative visual interpretation of these contrast-enhanced scans.

\subsection{Radiation treatment planning}

Radiation therapy was administered with 6,10 , or 18 megavolt photons to a standard field defined by the anatomic margins of the pre-operative breast: head of the clavicle, $2 \mathrm{~cm}$ below the inframammary fold, mid-sternum, and mid-axillary line. CT simulation was performed, and 3D planning was utilized. Standard fractionation with doses of 1.8-2 Gy per fraction was most common. Accelerated hypofractionation (2.66 Gy per fraction) was utilized in select cases. Tumor bed boost was used at the physician's discretion. Nodal radiation to axillary and supraclavicular lymph node basins was used when there was pathologic evidence of lymph node involvement. In the event of internal mammary lymph node involvement, a medial electron strip was utilized.

\subsection{Statistical analysis}

Continuous variables are expressed as mean $\pm \mathrm{SD}$ and frequencies or percentages for categorical variables which were calculated for all patient characteristics. Three groups of patients were compared by the testing procedure they received: mammogram only, CT scan only, or both. The groups were compared using analysis of variance (ANOVA) for continuous variables (i.e., age, lab values) and chi-square tests for categorical variables (i.e., cardiac risk factors). All effects were considered significant at $p$-value less than 0.05 . All the statistical analyses were performed with Statistical Analysis System (SAS) 9.4 software (Cary, NC, USA).

\section{Results}

\subsection{Baseline characteristics}

The baseline demographic and clinical characteristics of the patients are summarized in Table 1. One hundred and ninety $(74 \%)$ of them had follow up mammograms only, 34 (13\%) had chest CT scans only, and 32 (12\%) had both, mammograms and CT chest as follow up imaging modalities.

The mean follow-up duration of patients per individual imaging modality after initial radiation therapy is provided in Table 2. Breast cancer site and treatment modalities are listed in Table 3.

Variation in baseline characteristics, laboratory values, and incidence of co-morbidities across different imaging modalities is summarized in Supplementary Tables 1,2 .

The mean and mode values of total radiation dose administered per patient were 6167 and 6440 cGy respectively. The mode values of tangent and boost doses received
Table 1. Prevalence of baseline characteristics of all included patients.

\begin{tabular}{lc}
\hline Variable & Included patients $(\mathrm{N}=256)$ \\
\hline Mean age (y) & $60.7 \pm 13.4$ \\
Race & \\
-Caucasian (\%) & $183(71.5)$ \\
-African American (\%) & $51(19.9)$ \\
-Hispanic (\%) & $18(7)$ \\
Hypertension (\%) & $158(63.2)$ \\
Diabetes Mellitus (\%) & $66(26.4)$ \\
Dyslipidemia (\%) & $148(59.2)$ \\
Smoker (\%) & $95(38.6)$ \\
Prior documented CAD (\%) & $22(8.9)$ \\
Prior Myocardial infarction (\%) & $9(3.6)$ \\
Prior Heart Failure (\%) & $29(11.7)$ \\
\hline
\end{tabular}

*CAD, Coronary Artery Disease.

Table 2. Mean follow up duration from initial exposure to radiation therapy across different imaging modalities.

\begin{tabular}{lc}
\hline Imaging modality & Mean follow up duration in years \\
\hline All CT chest scans & $10.5 \pm 3.5$ \\
All Mammograms & $10.6 \pm 3.2$ \\
Mammogram only & $10.6 \pm 3.1$ \\
CT chest only & $9.5 \pm 3.6$ \\
CT chest and Mammogram & $11.5 \pm 3.2$ \\
\hline
\end{tabular}

Table 3. Breast cancer site and treatment modalities including surgery, chemotherapy and radiation therapy.

\begin{tabular}{lc}
\hline Breast cancer and treatment modalities & Included patients N =256 \\
\hline Cancer site & \\
-Right breast (\%) & $95(37.1)$ \\
-Left breast (\%) & $147(57.4)$ \\
-Bilateral (\%) & $14(5.5)$ \\
Mastectomy (\%) & $16(6.2)$ \\
Radiation therapy side & \\
-Right (\%) & $96(37.5)$ \\
-Left (\%) & $150(58.6)$ \\
-Bilateral (\%) & $10(3.9)$ \\
Chemotherapy (\%) & $173(74.2)$ \\
Cytotoxic therapy (\%) & $70(31.4)$ \\
Receptor therapy (\%) & $102(45.7)$ \\
Hormonal therapy (\%) & $139(62.3)$ \\
\hline
\end{tabular}

per patient were 5040 and 1400 cGy respectively. Minor variations in radiation dose across different imaging subsets is outlined in Supplementary Table 3.

Disparities in the laterality of breast cancer and treatment details amongst the patients in different imaging groups are listed in Supplementary Table 4. 
Significant proportion of the study group had dyslipidemia, which is a risk factor for atherosclerotic disease, that could be confounding the results. Excluding the $59.2 \%$ of patients with dyslipidemia and those with unknown dyslipidemia status, 19 patients with CT scans and 89 patients with mammograms at least 5 years after initial radiation therapy were analyzed independently as a subset of the total cohort.

\subsection{Computed tomography}

None of the 66 patients with CT scans, at least 5 years after initial radiation therapy, revealed IMA calcification or stenosis. One patient had known IMA atresia which was noted on a previous $\mathrm{CT}$ scan prior to receiving radiotherapy and was likely related to damage incurred during a surgical lymph node dissection.

Out of the patients that had post-radiation CT scans where the coronaries were assessed, 28 out of $66(42.4 \%)$ had coronary artery calcifications (CAC) present (Fig. 2). Of those 28 patients with CAC visualized on follow up CT scans, only 9 patients had pre-radiation CT scans available for comparison. Out of these 9 patients, only 2 (22.2\%) patients had no coronary calcification on pre-radiation imaging and therefore they were considered as new calcifications.

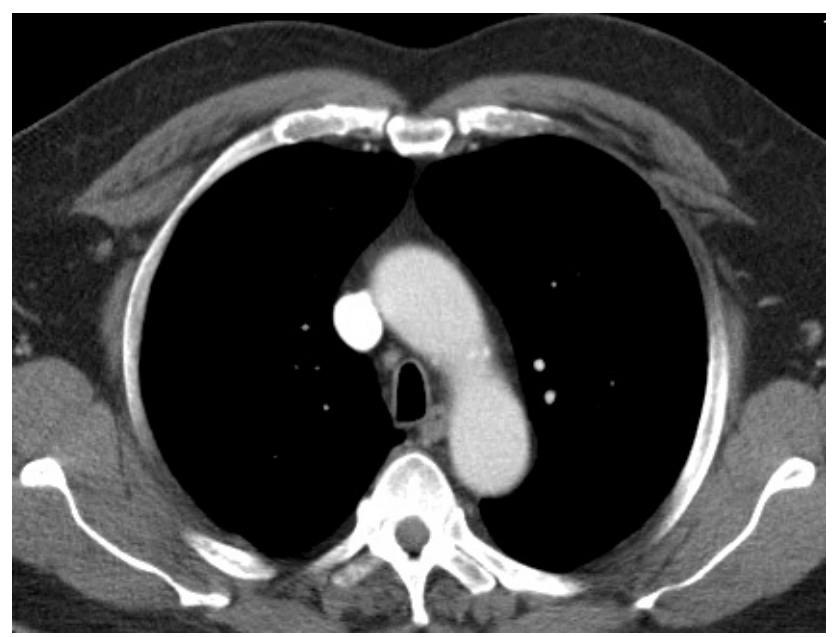

Fig. 2. A representative image of contrast enhanced CT scan showing patent IMAs bilaterally along the sternum.

Of the 28 patients who had CAC seen on follow up CT scans, only 4 (14.3\%) had mammogram findings of calcification on either side. There was no statistically significant association found between CAC and breast calcification found on mammograms ( $p=0.74)$ by Chi-square test.

None of the CT scans of the 19 patients without dyslipidemia revealed IMA calcification or stenosis. Ten of these patients had coronaries assessed on follow up CT scan and CAC was identified in 6 of them. Only 3 of the 6 patients had pre-radiation CT scans available for review and $\mathrm{CAC}$ was present in all three of them.

\subsection{Mammography}

A total of 222 patients had mammograms and 36 (16.2\%) were found to have calcifications (Fig. 3) on either side. Two hundred and ten patients received radiation to one side only. Further analysis of the mammogram data for the presence of calcifications in relation to radiation therapy revealed that 27 out of $210(12.9 \%)$ patients had calcifications on the side they received radiation therapy. Twenty-six of the $210(12.4 \%)$ patients had calcifications despite not receiving ipsilateral radiation therapy. Therefore, compared to the non-irradiated side, the odds of developing calcification on the radiated side were similar $(\mathrm{OR}=1.00)$.

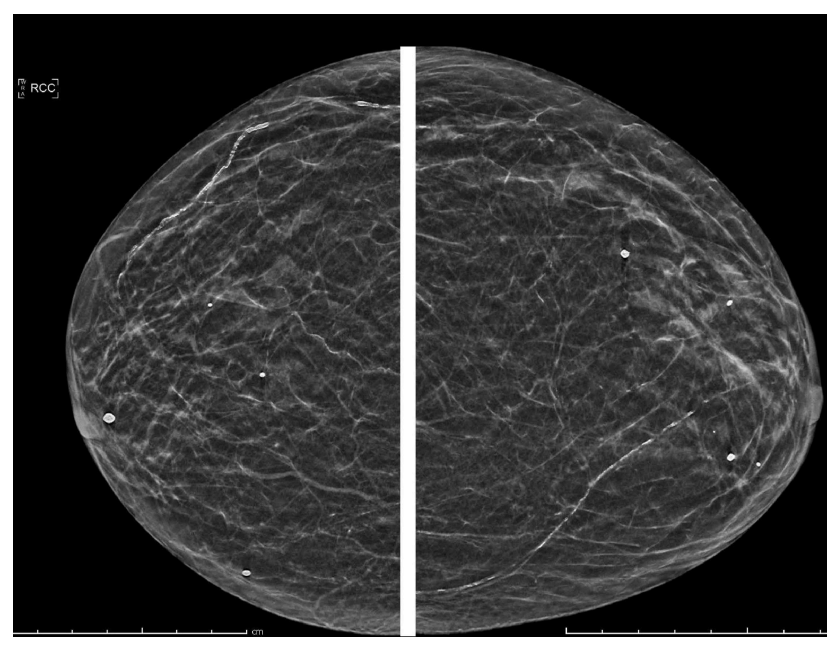

Fig. 3. Craniocaudal (CC) projection shows vascular calcifications in the inner aspect of the left breast, which represent calcified branches of the left internal mammary artery (IMA). Right breast CC view shows vascular calcifications in the outer aspect, which are not in the branches of IMA.

Among the 89 patients without dyslipidemia that had mammograms, $9(10.1 \%)$ were found to have calcifications on either side. Six of the $89(6.7 \%)$ patients had calcifications on the side they received radiation and an equal number of patients $(6.7 \%)$ had calcifications despite not receiving ipsilateral radiation therapy.

\section{Discussion}

Ischemic heart disease rates increase with exposure to radiation therapy among breast cancer patients $[1,11,12]$. The effect of radiation on IMA remains uncertain given insufficient radiographic data demonstrating vascular damage. Several studies showed concerns that radiation might increase IMA fragility, leading to early graft failure $[6,8,13$, 14]. On the other hand, IMA's have been historically recognized to have inherent protection against atherosclerosis compared to other arteries [15-17]. Using histologic analysis, recent studies reported no restrictions in the use of irradiated IMA's as grafts $[18,19]$. Other studies also used his- 
tomorphologic [19], and echocardiographic doppler investigations [20] to demonstrate that the IMA's are protected from radiation-induced damage [19]. Yet, a SCAI Expert Consensus Statement recommended using CCTA as an assessment tool for IMA's which could be affected by radiation therapy rendering it unfeasible as a graft [7].

This study showed that none of the patients with CT scans had new IMA calcifications, stenosis, or atresia at least 5 years after initial radiation therapy. The patient cohort represents a relatively intermediate-high risk cohort based on high rates of cardiac risk factors such as advanced age, hypertension, diabetes, dyslipidemia, and smoking history, despite them being of the female gender. As expected, based on this risk factor profile, CAC was seen in $42.4 \%$ of the patients. Yet, only $22 \%$ of the patients with coronary calcifications post-radiation were considered new calcifications. Given the small number of patients with preradiation $\mathrm{CT}$ scans, definitive conclusions as to the effect of radiation-induced atherosclerosis of the native coronary arteries in this cohort cannot be made. Nonetheless, the percentage of patients in this cohort with coronary calcification post-radiation is higher than what has been seen in prior studies with similar patient risk-profile [21]. The finding of new CAC on post-radiation CT scan compared to preradiation imaging was not observed in the small sub-set of patients without dyslipidemia, highlighting the influence of atherosclerotic risk factors, independent of radiotherapy on the study results. There was no statistically significant association found between CAC and breast calcifications found on mammograms. Finally, chest wall-irradiation was not associated with the development of vascular calcifications of the IMA branches when evaluated by mammography.

Study shortcomings such as inadequate radiation dose to cause IMA injury, insufficient follow-up duration, or less significant atherosclerotic risk profile of the patient cohort were considered as possible reasons for the absence of IMA damage. A cumulative dose of radiation $>3000$ cGy has been considered as the dose associated with a high risk for radiation-induced heart disease [22,23]. The mode value of the administered radiation dose in this study was $5040 \mathrm{cGy}$, and is higher compared to that reported in studies by Renner et al. [24] (3600 cGy), and Brown et al. [8] (3000-6000 cGy) which inferred that IMA's are susceptible to radiationinduced damage. In the studies reported by Gansera et al. [19] and Handa et al. [25] which concluded that there are no restrictions to the use of IMA conduits post-radiotherapy, the total dose reported was similar to the dose in the present study, i.e., 5000-6000 cGy. The mean duration from initial radiation exposure to IMA evaluation in this study was 10.5 years which is similar to that reported by Renner et al. [24] (11 years). The mean duration to the development of RICAD was reported as 9 years [26]. The prevalence of atherosclerotic risk factors in this study is comparable to that reported in a cohort of 25 patients by Brown et al. [8], which concluded that IMA graft patency in patients who received prior radiation therapy is less than expected for an arterial graft, and comparable to venous grafts. Compared to the patients in their study, the patient population in our study had a higher mean age in years (60 vs 56), a higher prevalence of hypertension ( $63 \%$ vs $52 \%)$, and diabetes mellitus (26\% vs $16 \%)$. From this data, it can be inferred that the patients in this study were exposed to a high enough dose of radiation, had an adequate long-term follow-up, and found to have a significant risk factor profile to observe IMA damage from radiation if it were to occur.

Several reasons may explain why none of the IMA's showed atherosclerotic disease on CT scan, (a) CT scans are insensitive to detecting radiation-induced IMA damage as an imaging modality, (b) IMA's are inherently protected against radiation-induced atherosclerosis, (c) Standard chest wall radiation does not result in sufficient IMA dose to produce detectable injury, (d) Longer follow up duration may be required. Although the diameter of the IMA's is smaller than that of the coronary arteries [27], multidetector CT angiograms have been studied to be adequate for the anatomical evaluation of the IMA's [27,28]. The latest published SCCT Appropriate Use Criteria [29] recommends cardiac $\mathrm{CT}$ to localize bypass grafts and other retrosternal anatomy before re-operative cardiothoracic surgery, and with the evolution of improved scanner technology, CT scan as a means to noninvasively assess the IMA's is being pursued [30]. However, there remains a paucity of data in the published literature regarding the utility of CT scan to evaluate for IMA calcification and stenosis.

There is conflicting data in the current literature as to whether the IMA's are inherently protected from the effects of radiation therapy $[6,13,14,19,20,24]$. Histomorphological analysis performed by Gansera et al. [19] studied 133 irradiated IMA's and compared them with a control group of 133 non-irradiated IMA's. Histomorphologic investigations did not identify severe fibrosis or radiation-induced damage of the IMA grafts. Nasso et al. [20] compared two groups of patients undergoing elective $\mathrm{CABG}$ with the use of IMA. There was no significant difference in intraoperative IMA flow when assessed by transthoracic echocardiography doppler between irradiated and non-irradiated individuals. Similarly, a retrospective clinical review by Handa et al. [25] of 47 patients undergoing CABG after mediastinal radiation therapy observed good early postoperative results without IMA graft failure. On the other hand, some studies suggest a higher risk of IMA graft failure after $\mathrm{CABG}$ in patients with prior radiation therapy $[6,8,13]$. The failure of IMA as a graft may be related to increased vessel friability and/or extensive mediastinal fibrosis resulting in intraoperative injury rather than radiation induced atherosclerosis [14,18,24]. This could explain the poor outcomes after CABG despite the absence of stenosis/calcification in IMA's.

The prevalence of calcifications identified on mammograms in the present patient population was $16 \%$. This is 
similar to the data published by Gunhan-Bilgen et al. [31], who observed a similar prevalence of breast calcification in post-radiation surveillance mammograms in breast cancer survivors. The prevalence of breast arterial calcification by mammogram in the general population ranges from $9 \%$ to $17 \%$ in women aged between 50-65 years and has been noted to be as high as up to $50 \%$ in older women [9]. As expected, the prevalence of calcifications on mammogram in the sub-set of patients without dyslipidemia is lower at $10 \%$ in comparison to the $16 \%$ observed in the entire cohort.

The clinical significance of calcifications seen on mammograms remains unclear. A statistically significant association between CAC and breast calcification was not observed. This is probably due to the small number of patients with CAC in our study. Breast artery calcification is known to be associated with the presence and even severity of CAC [32-34], but its diagnostic accuracy in predicting obstructive coronary artery disease is poor [33].

The precise anatomical site of breast calcification observed on mammograms can be difficult to localize. The compression of the breast tissue during the study itself can make it difficult to discern between the branches of IMA and other vasculature such as branches of the lateral mammary artery. There is also the possibility of macroscopic dystrophic/extravascular calcium deposition in the breast tissue which is not uncommon on screening mammograms [35]. Despite the calcifications seen on mammograms, no calcification of the IMA's was observed on the CT scans. The IMA branches may have different histological features compared to the IMA's, making them more susceptible to radiation-induced endothelial damage. In contrast to the IMA branches which run a more superficial course making them vulnerable to radiation, the IMA's run a relatively posterior course, perhaps shielded by the intercostal muscles and fascia.

\section{Conclusions}

This study provides a novel outlook in evaluating the radiographic assessment of IMA's with CT scans and mammograms in breast cancer patients exposed to chest wallradiation. To our knowledge, this is the first study to date to examine the utility of CT scans and mammograms in detecting IMA damage post-radiation therapy. Our study has some limitations. While this is a retrospective study conducted at a single center with small sample size, the strength of this analysis stems from the prospective, blinded examination of CT scans and mammograms by two independent radiologists. Our study included breast cancer patients only; this cohort was selected intentionally so that the effects of standardized radiation protocols could be compared. These findings, therefore, may not apply to other malignancies such as Hodgkin's Lymphoma that require a different dose and technique of radiation. The negative controls used were not healthy controls but rather the contralateral, non-irradiated sides. Although, this allows for equal baseline characteristics between the negative control and study sample, the absence of difference in calcifications between irradiated and non-irradiated sides in mammogram could have been potentially due to radiation scatter to the non-irradiated side. Our study however is hypothesisgenerating and future prospective studies with standardized imaging protocols (both chest CT and mammograms) should be entertained.

\section{Author contributions}

Concept and Design-WM, VN, SB, DG, AV; Research and Data Analysis-VN, WM, MA, MK, DBW, SB, AP, DG, DJH, AV; Manuscript PreAPration/Edit-VN, WM, MA, MK, DBW, SB, AP, DG, DJH, AV; Critical Revision Manuscript- $-\mathrm{VN}, \mathrm{WM}, \mathrm{SB}, \mathrm{DJH}, \mathrm{AV}$.

\section{Ethics approval and consent to participate}

Our study received the proper ethical oversight through our institutional IRB (SFH-18-110).

\section{Acknowledgment}

Not applicable.

\section{Funding}

This research received no external funding.

\section{Conflict of interest}

The authors declare no conflict of interest.

\section{Supplementary material}

Supplementary material associated with this article can be found, in the online version, at https://www.imrpre ss.com/journal/FBL/27/1/10.31083/j.fb12701030.

\section{References}

[1] Darby SC, Ewertz M, McGale P, Bennet AM, Blom-Goldman U, Brønnum D, et al. Risk of Ischemic Heart Disease in Women after Radiotherapy for Breast Cancer. New England Journal of Medicine. 2013; 368: 987-998.

[2] Soucy KG, Lim HK, Kim JH, Oh Y, Attarzadeh DO, Sevinc B, et al. HZE56Fe-Ion Irradiation Induces Endothelial Dysfunction in Rat Aorta: Role of Xanthine Oxidase. Radiation Research. 2011; 176: 474-485.

[3] Tungjai M, Whorton EB, Rithidech KN. Persistence of apoptosis and inflammatory responses in the heart and bone marrow of mice following whole-body exposure to ${ }^{28}$ Silicon $\left({ }^{28} \mathrm{Si}\right)$ ions. Radiation and Environmental Biophysics. 2013; 52: 339-350.

[4] Brosius FC, Waller BF, Roberts WC. Radiation heart disease. Analysis of 16 young (aged 15 to 33 years) necropsy patients who received over 3,500 rads to the heart. The American Journal of Medicine. 1981; 70: 519-530.

[5] Harskamp RE, Alexander JH, Ferguson TB, Hager R, Mack MJ, Englum B, et al. Frequency and Predictors of Internal Mammary Artery Graft Failure and Subsequent Clinical Outcomes: Insights from the Project of Ex-vivo Vein Graft Engineering via Transfection (PREVENT) IV Trial. Circulation. 2016; 133: 131-138. 
[6] Cuomo JR, Javaheri SP, Sharma GK, Kapoor D, Berman AE, Weintraub NL. How to prevent and manage radiation-induced coronary artery disease. Heart. 2018; 104: 1647-1653.

[7] Iliescu CA, Grines CL, Herrmann J. SCAI Expert consensus statement: Evaluation, management, and special considerations of cardio-oncology patients in the cardiac catheterization laboratory (endorsed by the cardiological society of india, and sociedad Latino Americana de Cardiologia intervencionista). Catheterization and Cardiovascular Interventions. 2016; 87: 895-899.

[8] Brown ML, Schaff HV, Sundt TM. Conduit choice for coronary artery bypass grafting after mediastinal radiation. The Journal of Thoracic and Cardiovascular Surgery. 2008; 136: 1167-1171.

[9] Reddy J, Son H, Smith SJ, Paultre F, Mosca L. Prevalence of breast arterial calcifications in an ethnically diverse population of women. Annals of Epidemiology. 2005; 15: 344-350.

[10] Jesinger RA. Breast anatomy for the interventionalist. Techniques in Vascular and Interventional Radiology. 2014; 17: 3-9.

[11] McGale P, Darby SC, Hall P, Adolfsson J, Bengtsson N, Bennet $\mathrm{AM}$, et al. Incidence of heart disease in 35,000 women treated with radiotherapy for breast cancer in Denmark and Sweden. Radiotherapy and Oncology. 2011; 100: 167-175.

[12] Boekel NB, Schaapveld M, Gietema JA, Russell NS, Poortmans $\mathrm{P}$, Theuws JCM, et al. Cardiovascular Disease Risk in a Large, Population-Based Cohort of Breast Cancer Survivors. International Journal of Radiation Oncology, Biology, Physics. 2016; 94: 1061-1072.

[13] Schulman HE, Korr KS, Myers TJ. Left internal thoracic artery graft occlusion following mediastinal radiation therapy. Chest. 1994; 105: 1881-1882.

[14] Hicks GL. Coronary artery operation in radiation-associated atherosclerosis: long-term follow-up. The Annals of Thoracic Surgery. 1992; 53: 670-674.

[15] Otsuka F, Yahagi K, Sakakura K, Virmani R. Why is the mammary artery so special and what protects it from atherosclerosis? Annals of Cardiothoracic Surgery. 2013; 2: 519-526.

[16] Sisto T. Atherosclerosis in internal mammary and related arteries. Scandinavian Journal of Thoracic and Cardiovascular Surgery. 1990; 24: 7-11.

[17] Barry MM, Foulon P, Touati G, Ledoux B, Sevestre H, Carmi $\mathrm{D}$, et al. Comparative histological and biometric study of the coronary, radial and left internal thoracic arteries. Surgical and Radiologic Anatomy. 2003; 25: 284-289.

[18] van Son JAM, Noyez L, van Asten WNJC. Use of internal mammary artery in myocardial revascularization after mediastinal irradiation. The Journal of Thoracic and Cardiovascular Surgery. 1992; 104: 1539-1544.

[19] Gansera B, Schmidtler F, Angelis I, Botzenhardt F, Schuster T, Kiask T, et al. Quality of internal thoracic artery grafts after mediastinal irradiation. The Annals of Thoracic Surgery. 2007; 84: 1479-1484.

[20] Nasso G, Canosa C, De Filippo CM, Modugno P, Mondugno P, Anselmi A, et al. Thoracic radiation therapy and suitability of internal thoracic arteries for myocardial revascularization. Chest. 2005; 128: 1587-1592.

[21] Takx RAP, Vliegenthart R, Schoepf UJ, Pilz LR, Schoenberg $\mathrm{SO}$, Morris $\mathrm{PB}$, et al. Coronary artery calcium in breast cancer survivors after radiation therapy. The International Journal of Cardiovascular Imaging. 2017; 33: 1425-1431.

[22] Lancellotti P, Nkomo VT, Badano LP, Bergler-Klein J, Bergler $\mathrm{J}$, Bogaert J, et al. Expert consensus for multi-modality imaging evaluation of cardiovascular complications of radiotherapy in adults: a report from the European Association of Cardiovascular Imaging and the American Society of Echocardiography.
Journal of the American Society of Echocardiography. 2013; 26: 1013-1032.

[23] Donnellan E, Phelan D, McCarthy CP, Collier P, Desai M, Griffin $\mathrm{B}$. Radiation-induced heart disease: a practical guide to diagnosis and management. Cleveland Clinic Journal of Medicine. 2016; 83: 914-922.

[24] Renner SM, Massel D, Moon BC. Mediastinal irradiation: a risk factor for atherosclerosis of the internal thoracic arteries. The Canadian Journal of Cardiology. 1999; 15: 597-600.

[25] Handa N, McGregor CG, Danielson GK, Orszulak TA, Mullany CJ, Daly RC, et al. Coronary artery bypass grafting in patients with previous mediastinal radiation therapy. The Journal of Thoracic and Cardiovascular Surgery. 1999; 117: 1136-1142.

[26] Hull MC, Morris CG, Pepine CJ, Mendenhall NP. Valvular dysfunction and carotid, subclavian, and coronary artery disease in survivors of hodgkin lymphoma treated with radiation therapy. Journal of the American Medical Association. 2003; 290: 28312837.

[27] Karaman B, Battal B, Bozkurt Y, Bozlar U, Demirkol S, Sahin MA, et al. The anatomic evaluation of the internal mammary artery using multidetector CT angiography. Diagnostic and Interventional Radiology. 2012; 18: 215-220.

[28] Tashima H, Fujikawa M, Izumi K, Matsuda K, Tomita K, Hosokawa K. Anatomic Evaluation of the Internal Mammary Vessels Using Multidetector CT Angiography Images in 100 Asian Patients. Eplasty. 2014; 14: e9.

[29] Taylor AJ, Cerqueira M, Hodgson JM, Mark D, Min J, O'Gara $\mathrm{P}$, et al. ACCF/SCCT/ACR/AHA/ASE/ASNC/NASCI/SCAI/SCMR 2010 Appropriate Use Criteria for Cardiac Computed Tomography. a Report of the American College of Cardiology Foundation Appropriate Use Criteria Task Force, the Society of Cardiovascular Computed Tomography, the American College of Radiology, the American Heart Association, the American Society of Echocardiography, the American Society of Nuclear Cardiology, the North American Society for Cardiovascular Imaging, the Society for Cardiovascular Angiography and Interventions, and the Society for Cardiovascular Magnetic Resonance. Journal of Cardiovascular Computed Tomography. 2010; 4: 407.e1-e33.

[30] Di Lazzaro D, Crusco F. CT angio for the evaluation of graft patency. Journal of Thoracic Disease. 2017; 9: S283-S288.

[31] Günhan-Bilgen I, Oktay A. Management of microcalcifications developing at the lumpectomy bed after conservative surgery and radiation therapy. AJR. American Journal of Roentgenology. 2007; 188: 393-398.

[32] Shah N, Chainani V, Delafontaine P, Abdo A, Lafferty J, Abi Rafeh N. Mammographically detectable breast arterial calcification and atherosclerosis. Cardiology in Review. 2014; 22: 69 78.

[33] McLenachan S, Camilleri F, Smith M, Newby DE, Williams MC. Breast arterial calcification on mammography and risk of coronary artery disease: a SCOT-HEART sub-study. Clinical Radiology. 2019; 74: 421-428.

[34] Maas AHEM, van der Schouw YT, Atsma F, Beijerinck D, Deurenberg JJM, Mali WPTM, et al. Breast arterial calcifications are correlated with subsequent development of coronary artery calcifications, but their aetiology is predominantly different. European Journal of Radiology. 2007; 63: 396-400.

[35] Joshi P, Sharma R. Benign Lesions on Screening Mammography: Increasing Diagnostic Confidence in a Hitherto Unscreened Population. Journal of Clinical and Diagnostic Research. 2017; 11: TC14-TC17. 